\title{
Construcción izada: Condicionantes estructurales del sistema REVERSTOP
}

\section{Lift building: Structural conditioning of REVERSTOP system}

\author{
Juan Pérez-Valcárcel $^{(*)}$, Manuel Muñoz-Vidal (**) ${ }^{(11 ́ c t o r ~ H e r m o ~}{ }^{(* * *)}$
}

\section{RESUMEN}

Un gran problema para la construcción de edificios es la construcción en altura, lo que supone un riesgo para los trabajadores y un mayor costo. El sistema REVERSTOP permite la construcción a nivel del suelo y el posterior izado de cada planta. El nivel superior es el primero en construirse en la planta baja. Este nivel se eleva a través de gatos hidráulicos y se construye un nuevo nivel debajo de él. Las fases posteriores permiten construir todo el edificio, siempre desde el nivel del suelo. En este artículo se analizan los aspectos estructurales del sistema. En el caso analizado, la estructura del edificio se compone de perfiles de aluminio que forman las paredes resistentes, los forjados y la cubierta, con el mismo elemento. Se aportan los cálculos que respaldan la efectividad de la solución y los resultados experimentales obtenidos en las pruebas realizadas dentro de un proyecto de investigación.

Palabras clave: Construcciones izadas, construcciones modulares, construcción inversa, construcciones ligeras.

\section{ABSTRACT}

A big problem for the construction of buildings is the construction in height, what supposes a risk for the workers and a greater cost. The patented REVERSTOP system allows the construction at ground level and the subsequent lifting of each plant. The upper level is the first to be built on the ground floor. This level is raised through hydraulic jacks and a new level is built under it. The subsequent phases allow building the entire building, always from the ground level. In this article we analyze the structural aspects of the system. In the case analyzed, the structure of the building consists of aluminum profiles that form the resistant walls, the floors and the roof, with the same element. The calculations that support the effectiveness of the solution and the experimental results obtained in the tests carried out within a research project are provided.

Keywords: Lifted constructions, modular constructions, top to bottom, lightweight constructions.

(*) Dr. Arquitecto. Catedrático de Estructuras. ETS Arquitectura, A Coruña (España).

(**) Dr. Arquitecto. Profesor Titular de Estructuras. ETS Arquitectura, A Coruña (España).

(**) Dr. Arquitecto. Profesor Contratado Doctor. ETS Arquitectura, A Coruña (España).

Persona de contacto/Corresponding author: valcarce@udc.es (Juan Pérez-Valcárcel)

ORCID: https://orcid.org/oooo-00o2-6440-5432 (Juan Pérez-Valcárcel); https://orcid.org/oooo-0003-0479-1279

(Manuel Muñoz-Vidal); https://orcid.org/oooo-00o3-0283-0852 (Víctor Hermo)

Cómo citar este artículo/Citation: Pérez-Valcárcel, Juan; Muñoz-Vidal, Manuel; Hermo, Víctor (2020). Construcción izada: Condicionantes estructurales del sistema REVERSTOP. Informes de la Construcción, 72(559): e355. https://doi.org/10.3989/ic.72993.

Copyright: (C) 2020 CSIC. Este es un artículo de acceso abierto distribuido bajo los términos de la licencia de uso y distribución Creative Commons Reconocimiento 4.0 Internacional (CC BY 4.0). 


\section{INTRODUCCIÓN}

Un problema recurrente en la construcción es el riesgo de trabajar a grandes alturas. Es un riesgo importante y creciente, en la medida que la mayor parte de las construcciones modernas tienen alturas que antes sólo aparecían en los grandes edificios monumentales. Además, la normativa de seguridad en la construcción es afortunadamente cada vez más estricta, lo que aumenta el interés de sistemas más seguros.

Por otra parte, la posibilidad de trabajar a nivel de suelo aporta gran facilidad en el traslado de materiales y herramientas lo que conlleva una mayor comodidad en el desarrollo del trabajo.

Relacionadas con el sistema constructivo que planteamos aquí, ya se han propuesto diversas soluciones, especialmente en el campo de las grandes cubiertas, algunas tan ingeniosas como el sistema Pantadome de M. Kawaguchi, que permite construir la mayor parte de la cubierta a nivel del suelo y luego izarla a su posición definitiva (1). Para edificios completos se han propuesto otro tipo de soluciones como las distintas variantes del sistema lift slab (2). Este sistema consiste en colocar los pilares del edifico con toda su altura, construir las placas a nivel del suelo y luego irlas izando a su posición definitiva por medio de gatos hidráulicos. Es un sistema relativamente barato y rápido, puesto que minimiza la necesidad de andamios y el hormigonado se hace en el suelo. Cuando la estructura está completamente construida, se ejecuta el resto del edificio, empezando por los cerramientos y particiones interiores.

Este último sistema tuvo un cierto desarrollo en la segunda mitad del siglo XX. Sin embargo, un grave accidente en L'Ambiance Plaza, un edificio de apartamentos en Bridgeport, Connecticut (USA), provocado por la inestabilidad en el izado, supuso un grave retroceso en el uso del sistema $(3,4,5)$ De hecho, el sistema de izado con gatos apenas se ha usado en edificación, aunque ha habido un interesante desarrollo en depósitos de chapa de acero izados por secciones (6).

Por otra parte, el sistema constructivo que se plantea en este artículo consiste en utilizar paneles de chapa metálica como elemento resistente principal. En un caso se trata de perfiles de aluminio extrusionados y en otro de chapas de acero plegadas. En ambos casos su comportamiento resistente es similar, puesto que ambas son secciones de pequeño espesor y gran esbeltez. Para este tipo de secciones existen numerosos estudios, puesto que suelen corresponder a la clase 4, según el Eurocódigo EC-3 (7). Esto supone que los elementos total o parcialmente comprimidos se abollan antes de alcanzar el límite elástico en la fibra más comprimida. Es un fenómeno complejo que ha sido objeto de numerosos estudios. Destacan los trabajos de Davies (8, 9, 10, 11), York (12), Veljkovic (13) o Craveiro (14).

Los sistemas constructivos a nivel de suelo basados en lift slab tienen dos serios problemas:

- Problemas de inestabilidad durante la construcción.

- No evita completamente el trabajo a gran altura. Sólo se elevan las losas por lo que el resto del trabajo debe realizarse en la losa cuando ya está elevada. El riesgo es menor, pero sigue existiendo.

Con todo, su ventaja principal, la construcción a nivel de suelo de edificios de varias plantas, sigue siendo un objetivo intere- sante. Por ello, nuestro equipo de investigación inició en 2011 una serie de trabajos para el diseño de un sistema simple y efectivo que permitiera construir edificios a nivel de suelo y posteriormente ir izando cada piso construido hasta su posición final. El elemento izado incluye forjados, cerramientos, particiones, instalaciones, acabados, etc. al contrario del lift slab.

La primera propuesta fue un sistema definido como CotaCero. Se trataba de diseñar un edificio con una estructura portante formada por muros resistentes de paneles de acero soldados entre sí. Con la adecuada distribución, los paneles resistentes pueden limitarse a los cerramientos exteriores y a las divisiones entre viviendas. El sistema es sencillo y eficaz y tiene unas prestaciones resistentes muy buenas. El principal problema es que la ejecución de las soldaduras continuas supone un importante coste y una ralentización de la obra. En 2012 nuestro equipo realizó un proyecto de investigación en el que se construyó un módulo completo, lo que nos permitió realizar una evaluación global y fiable de sus ventajas e inconvenientes. Los resultados de esta investigación se exponen en las referencias $(15,16,17 \mathrm{y} 18)$.

El principio básico del sistema CotaCero consiste en ejecutar cada planta del edificio a nivel de suelo para facilitar el trabajo. Luego se levanta por medio de gatos hidráulicos y se mantiene en posición hasta que se construye la placa inferior y se colocan los paneles perimetrales. Estos paneles son el elemento resistente principal. $\mathrm{Al}$ terminar de colocar los paneles, la losa superior queda ya sólidamente fijada. Entonces se bajan los gatos y se fijan a la losa inferior. En este momento ya se pueden construir los distintos elementos de esta planta en condiciones óptimas de seguridad. Cuando ya está totalmente ejecutada esta planta, se eleva el conjunto por medio de gatos. Reiterando este proceso puede levantarse un edificio de varias plantas trabajando siempre a nivel de suelo y por tanto en las mejores condiciones para los trabajadores, tanto en seguridad como en facilidad para trabajar.

\section{DEFINICIÓN DEL SISTEMA REVERSTOP}

\subsection{Aspectos generales}

Puesto que los resultados habían demostrado la viabilidad del sistema se inició un nuevo proyecto de investigación Reverstop $\AA$. Es una versión mejorada del CotaCero, manteniendo las mismas pautas. En este caso se trataba de diseñar nuevos paneles y juntas que permitieran una construcción con uniones atornilladas. Supone una mayor facilidad y rapidez en la ejecución y además es un sistema reversible. Todos los elementos del sistema son modulares y permiten el montaje y también el desmontaje y reutilización. Esto se fijó como objetivo básico de la investigación, tanto en los elementos resistentes como en los no estructurales. Como elementos resistentes se diseñaron los paneles de fachada, viguetas y suelos y como elementos no resistentes se diseñaron los paneles de suelo y los que recubren interiormente las fachadas. Estos paneles contienen las acometidas a las distintas instalaciones de fontanería, de saneamiento, de acondicionamiento térmico y de mecanismos eléctricos. El objetivo es que estos elementos vengan prefabricados de taller y puedan ser fácilmente colocados en obra. También permite una sencilla modificación del uso de los espacios, intercambiando los paneles.

Para poder estudiar la viabilidad del sistema se realizó un proyecto de investigación financiado por la Xunta de Galicia y con 


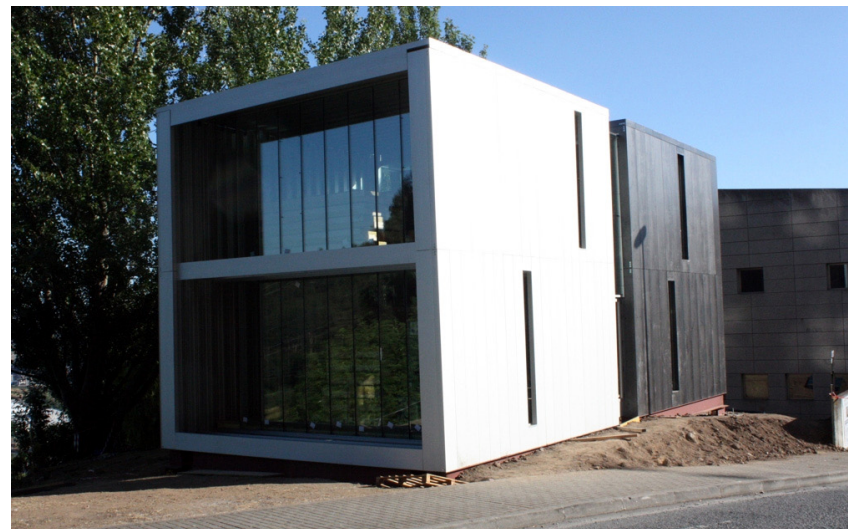

Figura 1. Conjunto de los dos módulos de Reverstop.

la colaboración de las empresas Instalacións Parcero S.L.U., ALUTEC y Talleres Formoso ${ }^{1}$. El proyecto se desarrolló entre febrero de 2014 y junio de 2015. Durante el proyecto se construyó en el Campus de A Zapateira de la Universidade da Coruña un edificio formado por dos módulos de 6x6 metros en planta con dos niveles con una altura total de $6 \mathrm{~m}$ (Figura 1). Uno de ellos se realizó con paneles de aluminio y el otro con paneles de chapa de acero, puesto que el proyecto incluía ambos materiales. Además, se colocó un módulo de enlace entre ambos con una escalera de acceso a la planta superior. En dicho módulo se colocaron paneles solares que proporcionaron la energía necesaria durante la fase experimental.

\subsection{Fases de la construcción con el sistema Revestop}

Las fases de construcción del sistema Reverstop se pueden resumir en los pasos desglosados en la figura 2.

Podemos describir ahora brevemente esos ocho pasos para hacernos una idea del esquema de funcionamiento de este sistema:

A. Se construye la cimentación por zapatas y una viga de atado perimetral (en color rojo). Se disponen los elementos auxiliares de izado: un gato hidráulico sobre cada zapata (en azul) y además dos vigas (morado). Estos elementos de izado se retiran al acabar el proceso.

B. Construcción del forjado de cubierta a nivel de suelo. Para ello se sitúan los paneles horizontalmente sobre las vigas de izado y se atornilla todo el conjunto.

C. Una vez montado y terminado el forjado de cubierta, los gatos de elevación pueden izar el conjunto a una altura correspondiente a una planta. La estructura queda sostenida únicamente por los gatos por lo que se debe realizar un arriostramiento provisional.

D. Se colocan los paneles de fachada, que se unen entre sí y a la cubierta.

E. Se bajan los gatos hidráulicos y se construye lo que será el suelo de la planta primera al nivel del terreno, que se une a los paneles de fachada. En este momento se puede construir a nivel de suelo todos los elementos interiores (tabiquería, instalaciones, etc) de la planta primera de la vivienda.

F. Nuevo izado de la parte construida a una altura correspondiente a una planta. De nuevo el conjunto está sostenido únicamente por los gatos por lo que vuelve a ser recomendable un arriostramiento provisional, máxime cuando ahora las
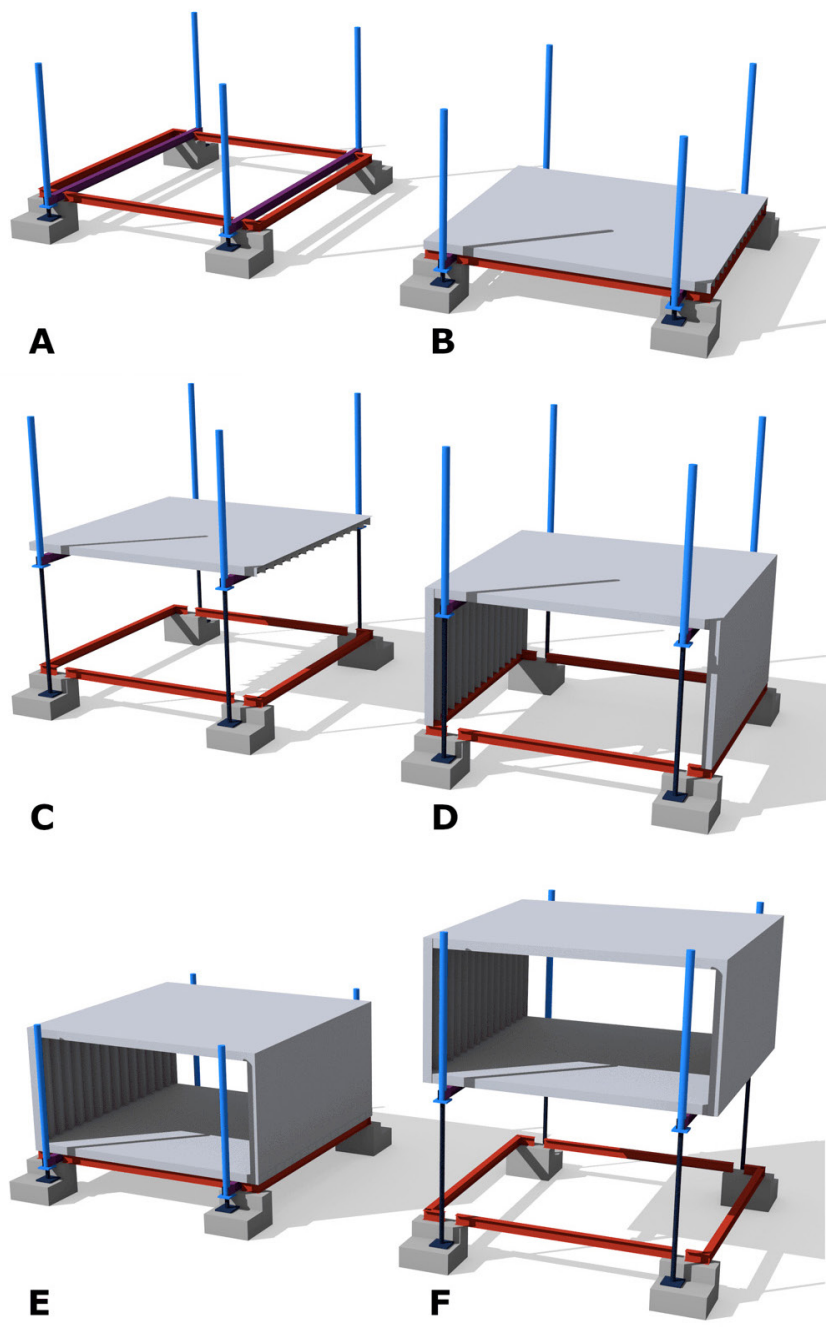

$\mathbf{E}$

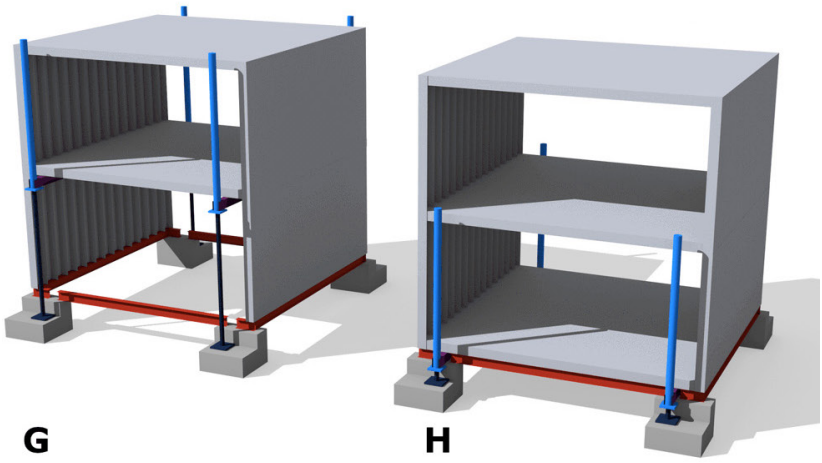

Figura 2. Proceso constructivo del sistema Reverstop.

cargas gravitatorias son mayores, y presenta una mayor superficie de incidencia del viento. Por cada planta que se eleve estas acciones irán aumentando, por lo que el arriostramiento provisional va cobrando cada vez mayor importancia.

G. Se construye el conjunto de paneles portantes de fachada de la planta baja que se unen al suelo de la planta primera.

$\mathrm{H}$. El edificio queda ya sustentado por los paneles de fachada, y entonces se pueden bajar los gatos y continuar con el montaje del forjado de la planta baja que quedará apoyado en la cimentación perimetral y unido a los paneles portantes de fachada de esta planta.

\footnotetext{
${ }^{1}$ Proyecto "Investigación e desenvolvemento dun novo sistema construtivo de crecemento modular reversible dende o nivel de terreo con criterios de eficiencia enerxética” IN852A 2014/12. Programa Conecta Peme. Xunta de Galicia.
} 
Este proceso se puede repetir tantas veces como plantas tenga el edificio. El único límite es el definido por la potencia de los gatos y la capacidad resistente de los paneles.

El sistema Reverstop permite trasladar a obra condiciones tipo taller y construir desde el nivel del terreno, como si de una cadena de montaje se tratase. Se logran así objetivos racionales como la calidad, rapidez o seguridad propia del taller.

\subsection{Sistema de izado}

Lo esencial del sistema consiste en construir cada planta a nivel del suelo y luego izarla hasta el nivel superior. A medida que el proceso transcurre la carga a izar y los esfuerzos horizontales son mayores. Esto implica problemas específicos que deben ser resueltos.

Un primer problema consiste en diseñar unos apoyos adecuados a fin de trasmitir a los gatos unos esfuerzos lo más centrados posible. Los gatos hidráulicos usados son capaces de resistir una carga de izado importante, pero los momentos deben ser trasmitidos a cimentación por medio de placas de anclaje. La unión no es totalmente rígida por lo que una posible carga excéntrica haría necesario usar chapas de gran espesor y fuertes anclajes. Además podría producir grandes desplazamientos laterales. El diseño de los apoyos debe evitar estos problemas.

Un aspecto a considerar son las condiciones de viento durante el izado. En condiciones normales suele fijarse como límite el habitual en estructuras móviles de $50 \mathrm{~km} / \mathrm{h}$, aunque es posible diseñar arriostramientos que permitan velocidades mayores.

Una vez izado el módulo permanece sujeto únicamente por los gatos hasta que se construye el nivel inferior de paneles portantes (figura 2f). Durante esta fase y por razones de seguridad se deben colocar arriostramientos capaces de garantizar la estabilidad del edificio. Para estructuras de pequeño tamaño, como el edificio citado es suficiente un arriostramiento formado por cables en X. Para estructuras de mayor tamaño puede utilizarse alguno de los sistemas que se proponen en la referencia (18).

\subsection{Juntas de apoyo}

Los paneles estructurales se apoyan sobre vigas de borde a través de un elemento junta. Para poder definir el material más adecuado se han realizado ensayos con diferentes materiales, como se describe en el apartado 3.3. Los materiales seleccionados fueron polietileno de alta densidad HDPE (High-density polyethylene), neopreno, etileno propileno dieno monómero EPDM (Ethylene Propylene Diene Monomer), goma reforzada con fibras de acero RNR y PVC reforzado con fibra de vidrio. Se ha considerado que el material más adecuado es el polietileno, que fue el usado en la construcción del prototipo.

\section{ELEMENTOS CONSTRUCTIVOS}

\subsection{Definición del módulo de aluminio}

El primer aspecto que se analizó fue el diseño de un perfil de aluminio capaz de resolver todos los requerimientos estructurales necesarios. Debía encajar entre los límites de la boquilla de extrusión y tener una resistencia suficiente tanto a compresión como a flexión en ambas direcciones.

El resultado de este análisis es el perfil es el que se indica en la figura 3. Colocado verticalmente forma una fachada resistente (1), colocado horizontalmente en la dirección de la inercia máxima se usa como vigueta (2) y horizontal pero en dirección de la inercia mínima forma la cubierta (3).

Cumpliendo todas las condiciones resistentes exigidas y considerando las dimensiones máximas que permite la boquilla de extrusión, el perfil es de la figura 4. Son muy características las dos entalladuras centrales, que permiten mejorar la resistencia a pandeo de la sección, evitando la abolladura.

En el caso del uso del perfil como viguetas, es necesario tener en cuenta que podría tener problemas de pandeo lateral. Por ello es necesario fijarlo al panel del suelo que sirve como elemento de arriostramiento. El esquema usado es el definido en la figura 5 .

\subsection{Definición del módulo de acero}

Los paneles del módulo de acero se construyeron con una chapa de acero de $3 \mathrm{~mm}$, plegada en la forma indicada en la figura. Al no haber problemas con la limitación de ancho de la boquilla de extrusión, como en el aluminio, se optó por unas dimensiones que permitieran un fácil montaje. El resultado es el perfil de la figura 6 .

En este caso se colocaron tres perfiles en U conformados en frío y soldados por puntos al perfil base. Estos perfiles permiten que la sección se comporte en clase 3, evitando abolladuras.

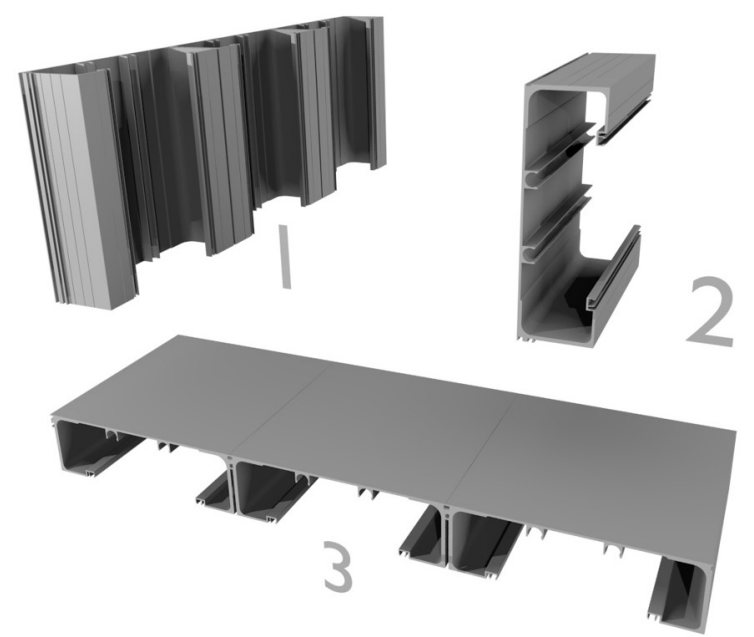

Figura 3. Perfiles de aluminio y su colocación en obra.

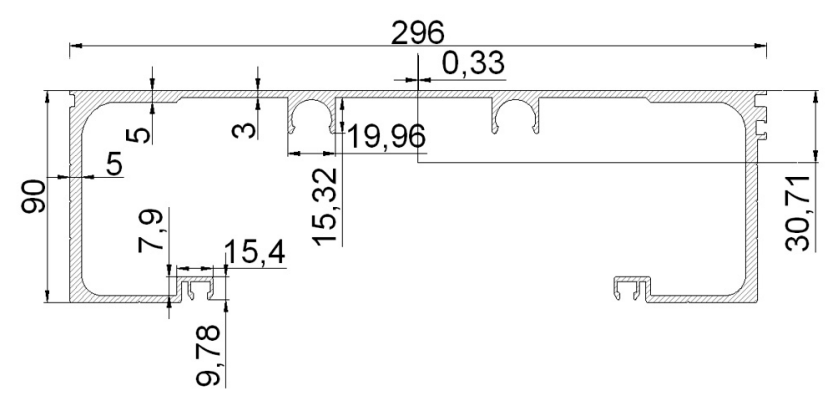

Figura 4. Perfil de aluminio. 


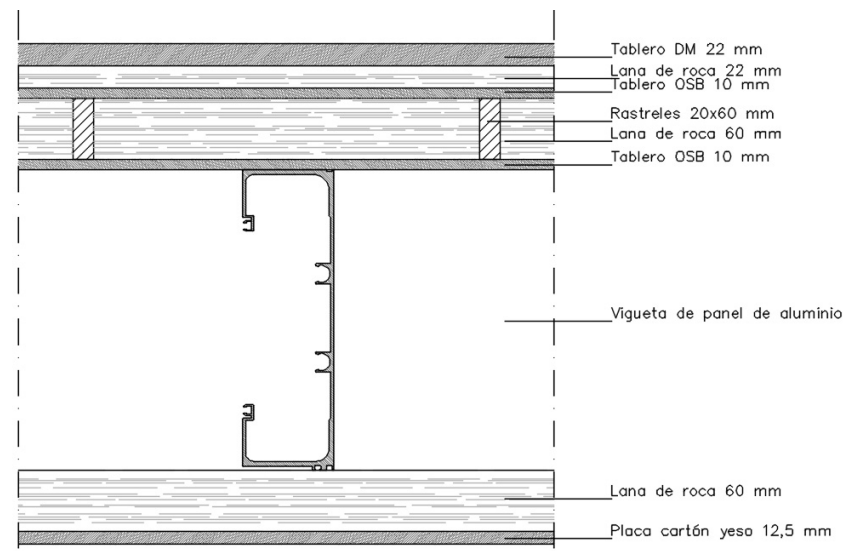

Figura 5. Composición del forjado.

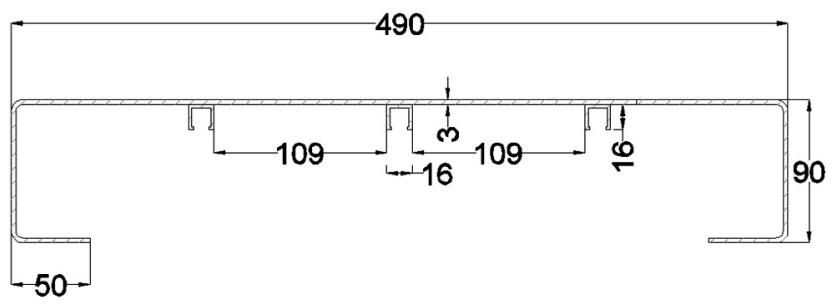

Figura 6. Perfil de acero.

Estos perfiles se atornillan entre sí formando la pared exterior resistente, en forma similar al módulo de aluminio. Los forjados se resuelven con perfiles laminados IPE-200, que se atornillan a los paneles de fachada.

\subsection{Definición de las juntas de apoyo}

Los perfiles resistentes deben trasmitir su carga a la viga de borde. No es posible el apoyo directo, sino que debe colocarse una junta capaz de resistir los esfuerzos trasmitidos y en su caso absorber las inevitables tolerancias de obra. La trasmisión directa a través de los tornillos no es efectiva, salvo en pequeñas edificaciones, puesto que las necesarias holguras de obra provocarían tensiones excesivas en los mismos. Al final todas las cargas de edificio se trasmitirían a los tornillos. En el módulo ensayado con luces de $6 \mathrm{~m}$ y dos plantas, la conexión entre paneles se hace por medio de aletas a las que se atornillan los paneles. Se pueden observar en las figuras 12 a 16. Sin embargo para luces y alturas mayores es preciso construir una viga perimetral sobre la que apoyan los paneles a través de juntas de HDPE. Esta será la solución estándar del sistema Reverstop.

A su vez esa viga trasmite su carga por compresión a los paneles inferiores. Puesto que no es posible conseguir una perfecta planeidad a las superficies de contacto, es necesario colocar igualmente una junta elástica intermedia.

Las prestaciones que debe satisfacer el material de la junta son.

- Debe permitir unas deformaciones plásticas suficientes para absorber las irregularidades de la junta.

- La junta no debe romperse bajo las tensiones aplicadas.

- Debe cumplir las condiciones de aislante térmico requeridas a todo el edificio.

Los paneles de fachada son los elementos resistentes de la estructura. Por ello las tensiones que trasmiten son bastante elevadas. Ningún material de junta puede resistir esos es- fuerzos en fase elástica: se trata de que puedan resistir esos esfuerzos, incluso con grandes deformaciones, pero sin llegar a romperse.

Los materiales seleccionados fueron HDPE, neopreno, EPDM, RNR (goma reforzada con fibras de acero) y PVC reforzado con fibra de vidrio. Sus características mecánicas son, considerando sólo valores característicos (Tabla 1):

Tabla 1. Características mecánicas del material de juntas.

\begin{tabular}{|l|c|c|c|c|c|}
\hline \multicolumn{1}{|c|}{ Material } & HDPE & Neopreno & EPDM & RNR & PVC \\
\hline Densidad $\left(\mathrm{g} / \mathrm{cm}^{3}\right)$ & 0.95 & 1.46 & 1.31 & 1.60 & 0.92 \\
\hline Lim. Elast. $\left(\mathrm{N} / \mathrm{mm}^{2}\right)$ & 23 & - & - & - & - \\
\hline Lim. rotura $\left(\mathrm{N} / \mathrm{mm}^{2}\right)$ & 37 & 9 & 7 & 4 & 50 \\
\hline E $\left(\mathrm{N} / \mathrm{mm}^{2}\right)$ & 800 & - & - & - & - \\
\hline Elongación (\%) & $>50$ & 250 & 300 & 200 & $>70$ \\
\hline
\end{tabular}

Se puede observar que únicamente en el caso del HDPE se puede aceptar un comportamiento razonablemente elástico. El resto de los materiales se deforman plásticamente, incluso a tensiones bajas, por lo que no podemos definir unas características mecánicas precisas, salvo la tensión de rotura. Para validar estos materiales se realizaron una serie de ensayos que se describirán en el apartado 4.3.

\section{ANÁLISIS ESTRUCTURAL}

\subsection{Análisis estructural de los perfiles de aluminio}

La estructura está formada por perfiles con la sección indicada que se atornillan entre sí para formar la fachada. Entre los paneles se dejan los huecos necesarios para la colocación de ventanas. Cada nivel de paneles se apoya sobre una viga de borde en acero que sirve para trasmitir las cargas y como elemento de apoyo en el izado de cada fase.

En la viga de borde se colocan unas cartelas a las que se unen las viguetas por medio de tornillos. Es una unión semirrígida suficiente para permitir una buena resistencia frente a esfuerzos horizontales. Los perfiles de cubierta se atornillan directamente a los paneles de fachada por medio de unas cartelas en $\mathrm{L}$, sin necesidad de viga de borde.

Para la ejecución de la estructura se ha empleado aluminio extrusionado con las siguientes características técnicas:

\begin{tabular}{|l|c|}
\hline Resistencia a rotura & $250 \mathrm{~N} / \mathrm{mm}^{2}$ \\
\hline Ductilidad alargamiento & $15 \%$ \\
\hline Módulo elástico & $69000 \mathrm{MPa}$ \\
\hline Densidad & $2700 \mathrm{Kg} / \mathrm{m}^{3}$ \\
\hline Punto de fusión & $660^{\circ} \mathrm{C}$ \\
\hline Intervalo de temperatura de trabajo & $-250 / 150^{\circ} \mathrm{C}$ \\
\hline Conductividad eléctrica & $29 \mathrm{~m} / \mathrm{Ohm} \cdot \mathrm{mm}^{2}$ \\
\hline Conductividad térmica & $200 \mathrm{w} / \mathrm{m}^{\circ} \mathrm{C}$ \\
\hline Coeficiente dilatación lineal & $2410-6 /{ }^{\circ} \mathrm{C}$ \\
\hline Magnético & $\mathrm{No}$ \\
\hline Soldable & $\mathrm{Si}$ \\
\hline
\end{tabular}

En general el cálculo estructural no presenta dificultades especiales, pero existen dudas en cuanto a su comportamiento a pandeo. Para ello se realizaron estudios teóricos y por el MEF y también se realizaron ensayos de compresión hasta 
llegar al colapso (Figura 7), que confirmaron las hipótesis iniciales. Los cálculos en ordenador se realizaron con el programa AutoFem.

Los ensayos se realizaron en el laboratorio de PEMADE (USC) utilizando una prensa modelo $\mathrm{SMH} / 4 / \mathrm{SCM} / 2$ de Microtest con una capacidad máxima de carga de $600 \mathrm{kN}$ y con un recorrido máximo de $3 \mathrm{~m}$. Para conseguir apoyos articulados se utilizaron rótulas esféricas diseñadas para ensayos de compresión por el Grupo de Estructuras Arquitectónicas de la universidad de A Coruña (19). Los resultados de los ensayos fueron (Tabla 2):

Tabla 2. Resultados de los ensayos a compresión.

\begin{tabular}{|l|c|}
\hline Ensayo perfil 1 & $152,29 \mathrm{kN}$ \\
\hline Ensayo perfil 2 & $161,75 \mathrm{kN}$ \\
\hline Ensayo perfil 3 & $167,12 \mathrm{kN}$ \\
\hline
\end{tabular}

Para realizar el ensayo las piezas de apoyo formaban la rótula en el centro de la sección que no coincide con el centro de gravedad. La carga tiene una excentricidad de $15.23 \mathrm{~mm}$, que debe ser considerada en los cálculos.

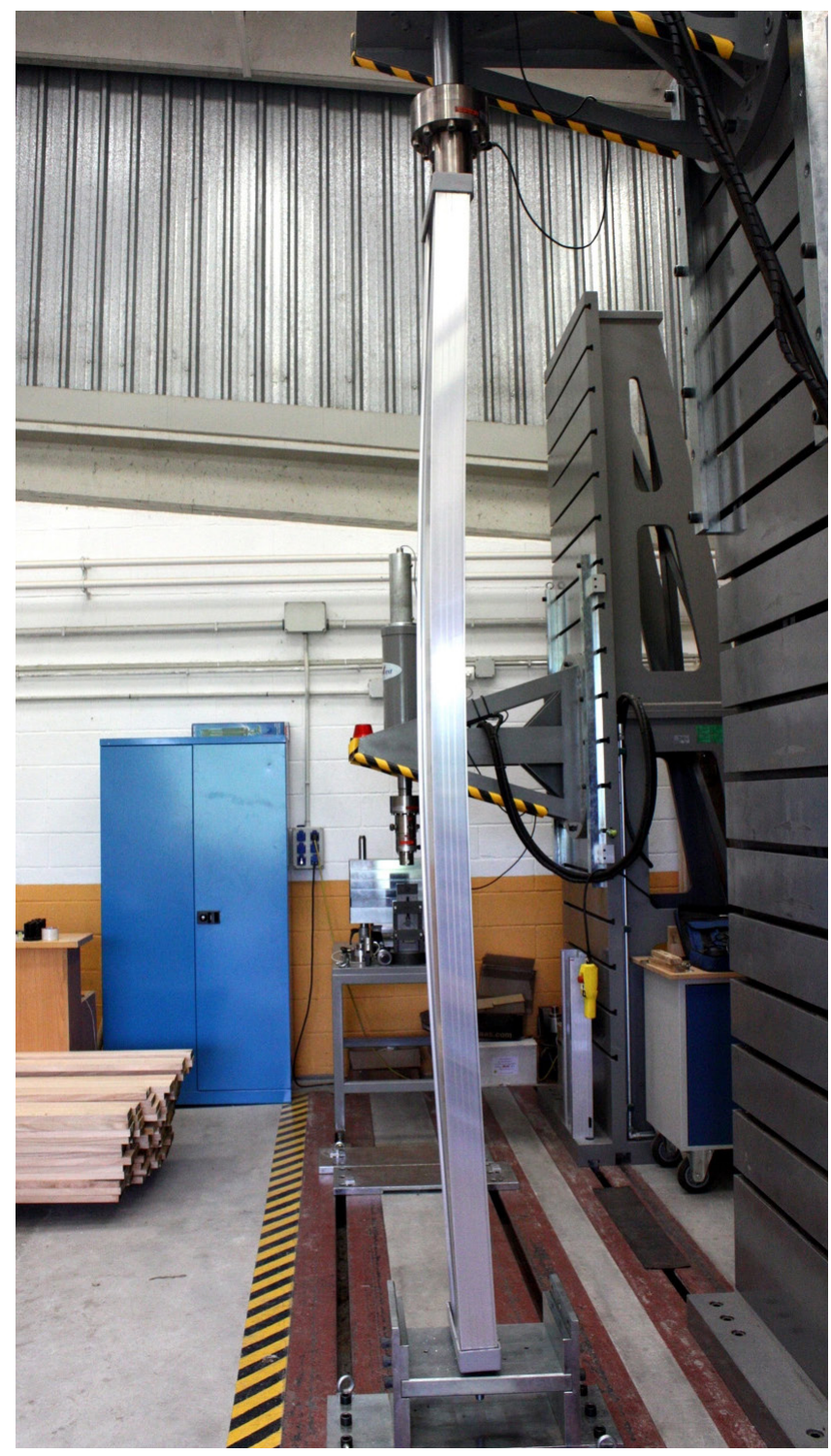

Figura 7. Ensayo a compresión del perfil de aluminio.
El momento máximo considerando una barra articulada en sus extremos y cálculo de segundo orden es [1]

$$
M_{\max }=P \cdot e \cdot \sec \frac{p \cdot 1}{2} \text { siendo } p=\sqrt{\frac{P}{E \cdot I_{x}}}
$$

Con los datos del perfil la tensión límite de 250 Mpa se produce para una carga aplicada con la excentricidad real de $153.49 \mathrm{kN}$. Se consideró que la coincidencia de resultados fue muy buena por lo que se estimó innecesario realizar una campaña más amplia. Por otra parte, estos resultados validan los modelos teóricos, confirmados por el MEF por lo que el resto de las comprobaciones se realizaron con cálculos en ordenador.

\subsection{Análisis estructural de los perfiles de acero}

Para la ejecución de la estructura se ha empleado acero S 275 JR en chapa de $3 \mathrm{~mm}$ cortada y doblada según el esquema indicado. El acero tiene las siguientes características técnicas:

\begin{tabular}{|l|c|}
\hline Resistencia a rotura & $410 \mathrm{~N} / \mathrm{mm}^{2}$ \\
\hline Límite elástico $(\mathrm{t}=3 \mathrm{~mm})$ & $275 \mathrm{~N} / \mathrm{mm}^{2}$ \\
\hline Módulo elástico & $210000 \mathrm{MPa}$ \\
\hline
\end{tabular}

En este caso se ha estimado que no era necesario un refrendo experimental, por la alta fiabilidad del acero. Además los esfuerzos previstos son muy inferiores a su capacidad resistente. Por ello todos los cálculos se han realizado con modelos teóricos, contrastados con cálculos numéricos con el MEF.

\subsection{Análisis experimental de las juntas}

Para ensayar las juntas en condiciones similares a las del edificio final, se diseñó un banco de pruebas específico para este ensayo. En la figura 8 se muestran las características del montaje.

Los ensayos se realizaron en el laboratorio de la EUAT de la Universidade da Coruña, utilizando una máquina de ensayo a compresión Omadisa 120-31 con una célula de carga Microtest $\mathrm{PBI} / 20$ con control digital de gran precisión. Admite una carga máxima de $200 \mathrm{kN}$. Los ensayos se realizaron para una tensión máxima de cálculo de aproximadamente $100 \mathrm{Mpa}$ que es más del doble de la prevista en el edificio real, que era de 42,53 Mpa en la situación más desfavorable. Esto supuso que la carga máxima a aplicar en el ensayo fue de $185 \mathrm{kN}$. Con estos ensayos se intentaba disponer de datos para intentar aumentar el número de plantas o disminuir el espesor del panel.

La puesta en carga para estos ensayos fue a una velocidad de $200 \mathrm{~N} / \mathrm{s}$ hasta llegar a la carga fijada. El intervalo de muestreo fue de 1 segundo, y número de lecturas por ensayo fue del orden de un millar.

En primer lugar se realizó una serie de seis ensayos para cada uno de los materiales. En esta primera serie se colocó la muestra de junta sobre el perfil inferior, sobre ella se colocó un conjunto de dos medios paneles de acero y la carga se aplicó por medio de un perfil superior. El punto de aplicación de la carga se hizo coincidir con el c.d.g. del conjunto de ambos medios paneles. 


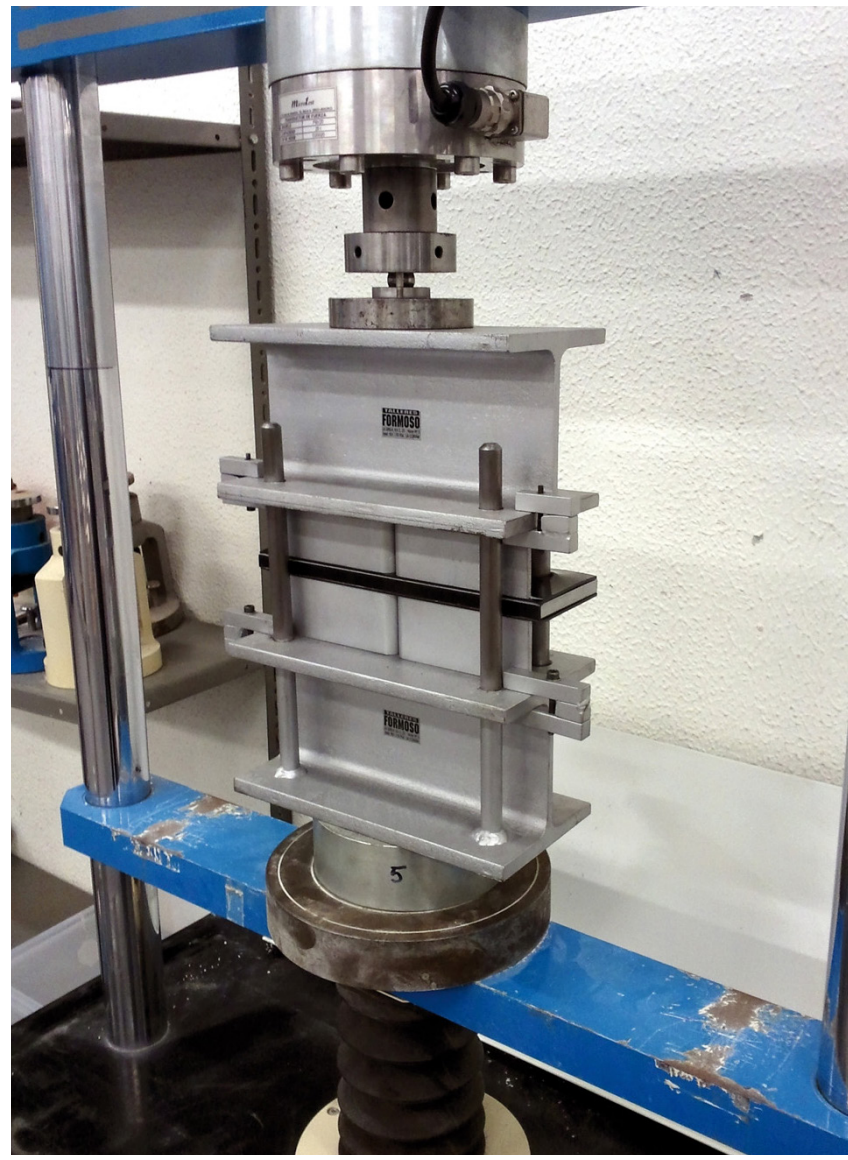

Figura 8. Ensayo del material de las juntas.

La prensa proporciona los datos de carga trasmitida y desplazamiento medido. Es necesario corregir los resultados de desplazamiento, porque también se produce un acortamiento elástico del banco de prueba. Para ello se realizó un conjunto de seis ensayos sin junta. Las cargas se trasmitían directamente a través de los paneles de acero y con ello se pudo medir el desplazamiento sin considerar el efecto de la junta. Los resultados fueron muy coincidentes, por lo que este valor pudo restarse del los resultados de los ensayos para obtener directamente los desplazamientos del material de la junta.

Estas primeras series de ensayos permitieron constatar que el único material que era capaz de resistir la carga total aplicada sin romperse era el HDPE. Sin embargo existía la duda de cuál podría ser su comportamiento en las condiciones reales del edificio. Por ello se programó una nueva serie de ensayos, que esta vez se concentraron en juntas de HDPE, puesto que las demás ya habían sido descartadas. Los resultados de las segundas series de ensayos se exponen en la figura 9.

En las gráficas se indican los resultados de cada uno de los ensayos realizados como PE1 a PE5. Se observa que tienen poca variabilidad lo que indica un comportamiento bastante uniforme de las chapas de HDPE. También se indica el valor PE medio y el resultado del ensayo en vacío que permite conocer el acortamiento elástico real del montaje. Restando al comportamiento medio el valor del desplazamiento en vacío, podemos obtener con buena precisión el comportamiento elasto-plástico de la junta de HDPE. Este resultado se muestra en la línea de color rojo.

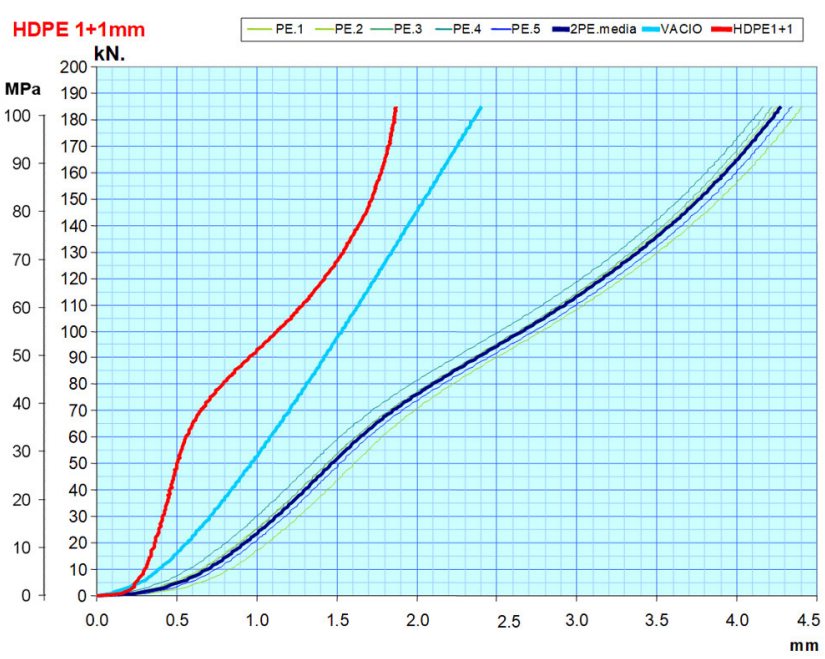

Figura 9. Resultados de los ensayos en juntas de HDPE.

Tabla 3. Resultados ensayos a largo plazo.

\begin{tabular}{|l|c|c|c|c|c|c|}
\hline \multicolumn{1}{|c|}{ Semanas } & $\mathbf{0}$ & $\mathbf{1}$ & $\mathbf{2}$ & $\mathbf{3}$ & $\mathbf{4}$ & $\mathbf{1 2}$ \\
\hline HDPE $(1 \mathrm{~mm})$ & 0.75 & 0.25 & 0.20 & 0.15 & 0.15 & 0.15 \\
\hline HDPE $(2 \mathrm{~mm})$ & 1.30 & 0.45 & 0.40 & 0.35 & 0.30 & 0.30 \\
\hline
\end{tabular}

Puesto que estos ensayos se realizaron a corto plazo se realizó un ensayo a largo plazo para analizar el comportamiento de la junta de HDPE. Para ello se construyó un modelo simple de forma que se pudiera aplicar una carga de $714 \mathrm{~N}$ sobre una zona de 30x5 mm en una serie de probetas de 60x30 $\mathrm{mm}$ de HDPE de dos espesores diferentes 1 y $2 \mathrm{~mm}$. La tensión aplicada fue de 47,5 MPa. La carga se aplicó a distintas probetas con diferentes intervalos temporales y el espesor final se midió con un calibre modelo Metrica de Dickenmessgerät con una presión de $0,05 \mathrm{~mm}$. Los resultados se muestran en la tabla 3 .

Estos ensayos permitieron constatar la eficacia de la junta de HDPE. Incluso para tensiones muy altas, las probetas sufrieron grandes deformaciones, pero en ningún caso se produjo la rotura, confirmando los resultados de los ensayos a corto plazo.

\subsection{Análisis estructural del edificio completo}

El esquema estructural del edificio está basado en un sistema de muros portantes formado por perfiles atornillados entre sí. Estos perfiles se apoyan en el borde superior y el inferior en vigas de borde, a las que se unen las viguetas de forjado. Esta disposición permite construir plantas de diversas formas. En el prototipo construido uno de los bloques estaba formado por perfiles de aluminio y el otro de perfiles de acero. En ambos casos los forjados estaban apoyados sobre dos paredes resistentes, siendo las demás simples cerramientos.

En ambos casos las viguetas de forjado estaban calculadas para luces de hasta 7,20 m, aunque el modelo se construyó con $6 \mathrm{~m}$ de luz. En el caso de las viguetas de aluminio se colocó el panel de suelo atornillado al ala superior para actuar como arriostramiento frente a posibles problemas de pandeo lateral. En el caso de las viguetas de acero este riesgo no existía, por lo que los paneles de suelo se colocaron sobre las viguetas sin necesidad de fijación. 


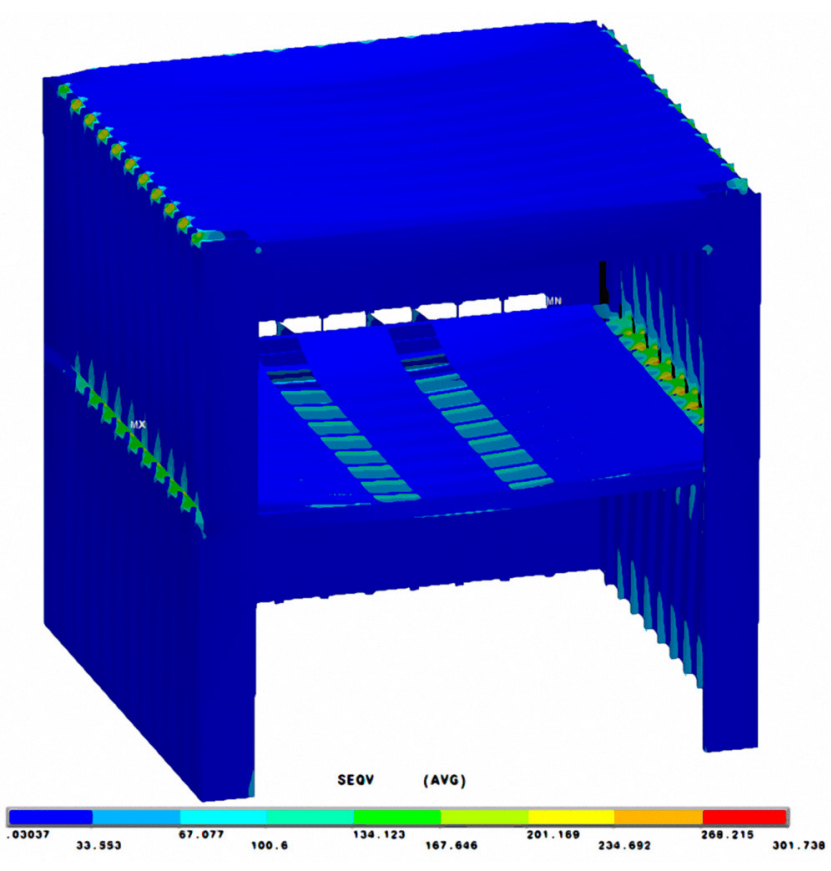

Figura 10. Cálculo del módulo de aluminio.

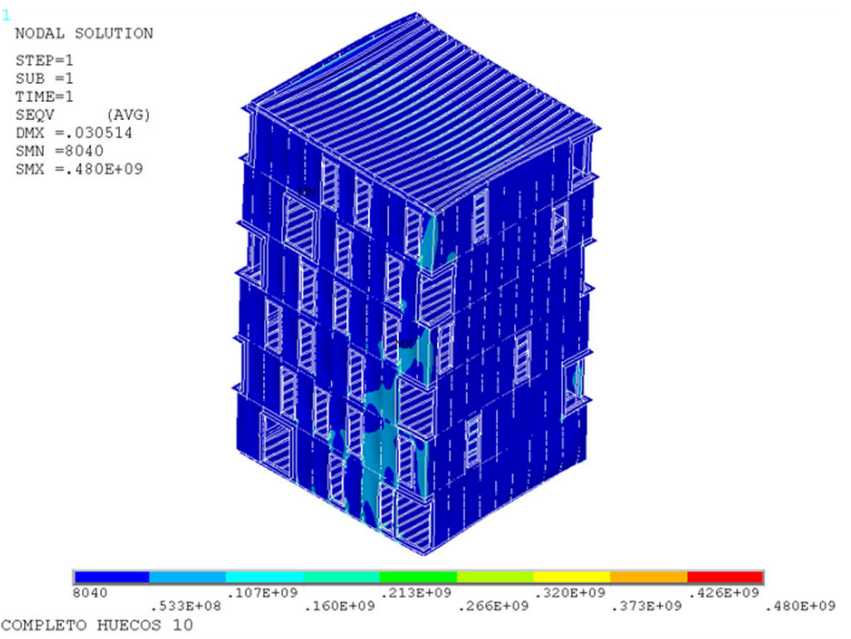

Figura 11. Cálculo de un edificio de 6 plantas.

Los cálculos efectuados son:

- Estabilidad del conjunto en las fases de construcción e izado.

- Peso propio incluyendo la tabiquería.

- Acciones variables: Sobrecarga de uso, viento y nieve.

- Acciones sísmicas.

- Acciones térmicas.

Todos estos cálculos se han efectuado según la normativa española (Código Técnico de la Edificación) que exige unas prestaciones similares a las de los Eurocódigos. Se han analizado tanto los ELU como los ELS (Figura 10).

En el modelo de cálculo se han utilizado distintas variantes considerando los huecos de fachada. La disposición de estos huecos no necesita ser regular lo que aporta mayor flexibilidad al diseño.

Para poder tener en cuenta las posibles situaciones de pandeo se ha utilizado el método de elementos finitos en cálculo no lineal.
Los resultados obtenidos muestran que con los perfiles utilizados pueden resolverse sin problemas edificios de hasta seis plantas. Para edificios de más altura pueden utilizarse paneles de mayor espesor o emplear paneles resistentes en las divisiones interiores. Sin embargo consideramos que este sistema es más efectivo y recomendable para alturas de hasta 6 plantas (Figura 11).

\section{CONSTRUCCIÓN DEL PROTOTIPO}

\subsection{Construcción del módulo de aluminio}

El primer módulo en ser construido fue el de aluminio, al ser el más novedoso y ligero. Por otra parte, el sistema es idéntico en el caso del módulo de acero. Los problemas de diseño, construcción e izado son idénticos en ambos casos.

Además de resolver la construcción de ambos módulos se construyeron los paneles que forman la cara interior de las paredes y los suelos. También se construyeron diversos dispositivos de instalaciones pasivas y se ensayaron en el transcurso de la investigación. El presente artículo se centra en los aspectos estructurales.

El edificio se mantuvo construido hasta el año 2016 (figura 1). Durante este período se realizaron diversos ensayos de acondicionamiento, aislamiento y otras condiciones funcionales. También se analizó la durabilidad de los elementos estructurales. Luego el edificio se desmontó, fue retirado por las empresas constructoras y sus elementos reutilizados. Se han construido diversos edificios y se prevé la construcción de un pabellón de exposiciones este año con una variante mejorada del perfil de aluminio.

\subsection{Fases de la construcción de los módulos}

Las fases de construcción de cada módulo han sido las siguientes:

Construcción de la base de apoyo (figura 12). En primer lugar se colocaron zapatas prefabricadas con un plano superior coincidente con la cota del terreno y superficie de trabajo. Estaban calculadas para los esfuerzos finales y los de todas las fases del proceso de izado. Luego se colocó sobre ellas un emparrillado de vigas de acero IPE-330 sobre las zapatas para servir de apoyo a los muros resistentes.

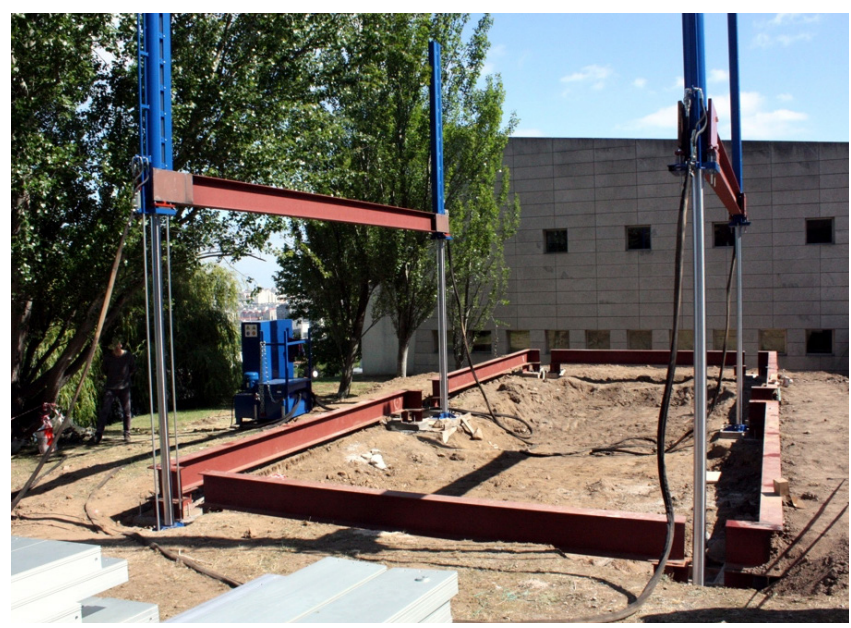

Figura 12. Construcción de la base de apoyo. 
Colocación de los gatos hidráulicos. Se han utilizado sólo cuatro gatos del tipo MDI Ø125 / Ø90х3000 BD de Kraken Ingeniería Técnica S.L. con un recorrido de 3,00 m y una capacidad de carga de $400 \mathrm{kN}$. Con los cuatro gatos disponibles se izó primero el módulo de aluminio y posteriormente los gatos se cambiaron de sitio para izar el módulo de acero.

Colocación de las vigas de izado sobre los gatos. Estas vigas están formadas por perfiles IPE-300 y disponen de dos cartelas laterales de $300 \mathrm{~mm}$ de canto y $30 \mathrm{~mm}$ de espesor para evitar movimientos en la cabeza de los gatos. Sobre ellas se colocaron las viguetas de cada planta. En este caso no fue necesario prever dispositivos para permitir movimientos laterales sobre su eje vertical para permitir los movimientos necesarios.

Construcción de la planta de cubierta (figura 13). Para ello se situaron los paneles horizontalmente sobre las vigas de izado y se atornillaron. En este caso fue necesaria una ejecución especialmente cuidadosa puesto que los propios perfiles se utilizaron como cubierta, sin ningún tipo elemento de impermeabilización añadido. Una vez que el forjado de la cubierta estuvo totalmente construido, se realizaron comprobaciones de estanqueidad inundando la cubierta.

Izado de la cubierta (figura 14). A continuación se procedió a izar el conjunto de la cubierta completo. Para evitar problemas de inestabilidad se colocaron cables en forma de aspa que quedarían tensos a alcanzar el máximo nivel de izado. En condiciones normales es una precaución razonable, pero en este caso no se produjo ninguna situación que exigiera la

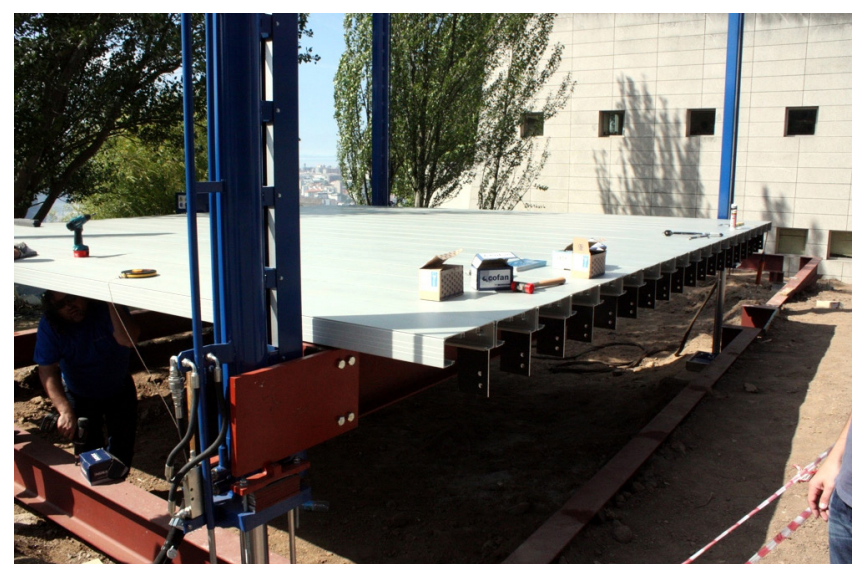

Figura 13. Planta de cubierta sobre gatos para su izado.

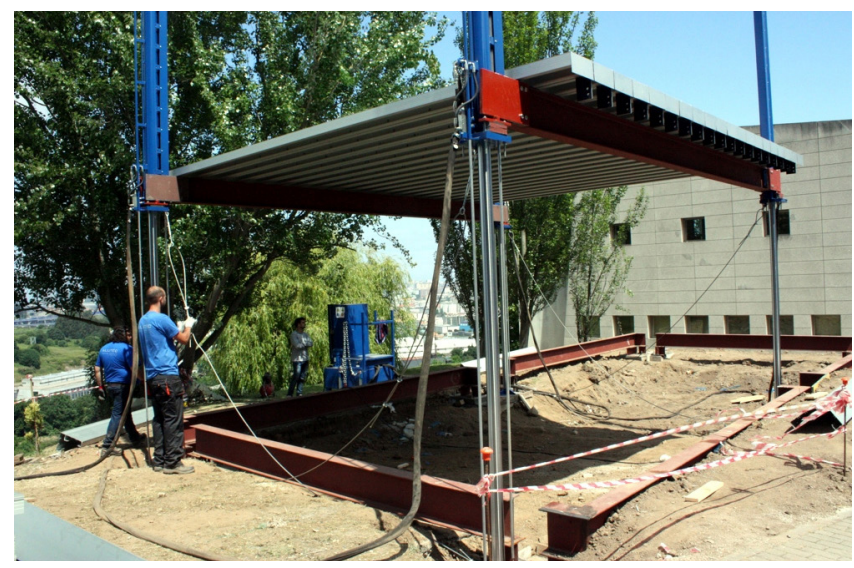

Figura 14. Izado de la cubierta. puesta en carga de los cables, ni siquiera en situaciones del viento de cierta intensidad. Los gatos de elevación, que sujetan las vigas de izado, elevaron el conjunto a una altura correspondiente a una planta $(3 \mathrm{~m})$.

Colocación de los paneles de fachada (figura 15). A continuación se colocaron los paneles apoyados en el emparrillado inferior. Los paneles se fijaron con tornillos a la cubierta y entre sí por medio de cartelas de aluminio. Entre los paneles se colocaron juntas de HDPE.

Construcción del forjado inferior y acabado de la planta (figura 16). Una vez montados los paneles de fachada, el conjunto es autoportante. Ya se pueden hacer descender los gatos de elevación situando las vigas de izado a la cota adecuada para montar el forjado inferior apoyado sobre ellas. Una vez solidarizado el forjado, comenzó el montaje de paneles de suelo, y de los huecos reforzados con sus carpinterías, hasta configurar una sección de fachada cerrada. También se pueden construir a nivel de suelo todos los elementos interiores de la vivienda.

Izado de la parte construida (figura 17). A continuación se izó todo el conjunto del piso superior. Nuevamente se colocaron cables de arriostramiento, que como en el caso anterior no llegaron a entrar en carga.

Con la planta superior en su posición definitiva, se montaron los paneles resistentes de la planta inmediatamente inferior. Nuevamente el edificio quedó estabilizado y ya se pudieron bajar los gatos para continuar el proceso. En este caso como

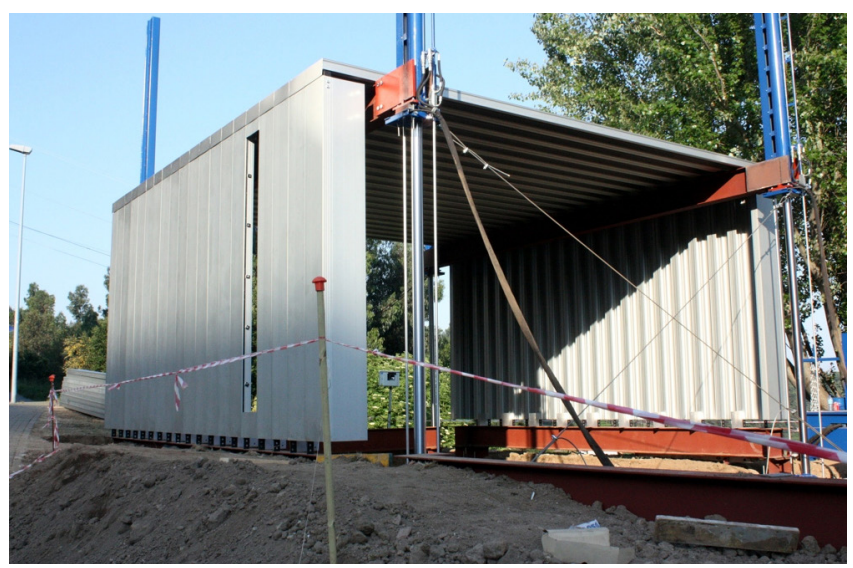

Figura 15. Colocación de los paneles de fachada.

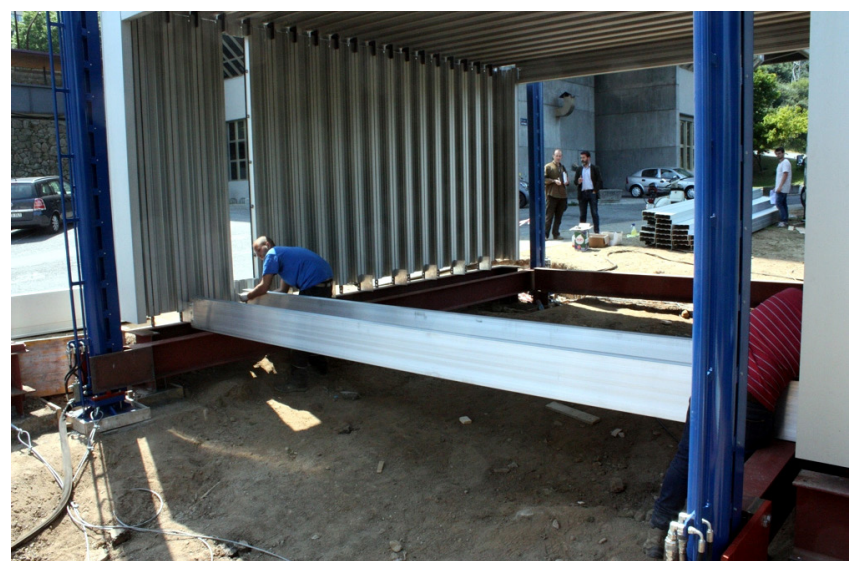

Figura 16. Construcción del suelo de la planta superior. 


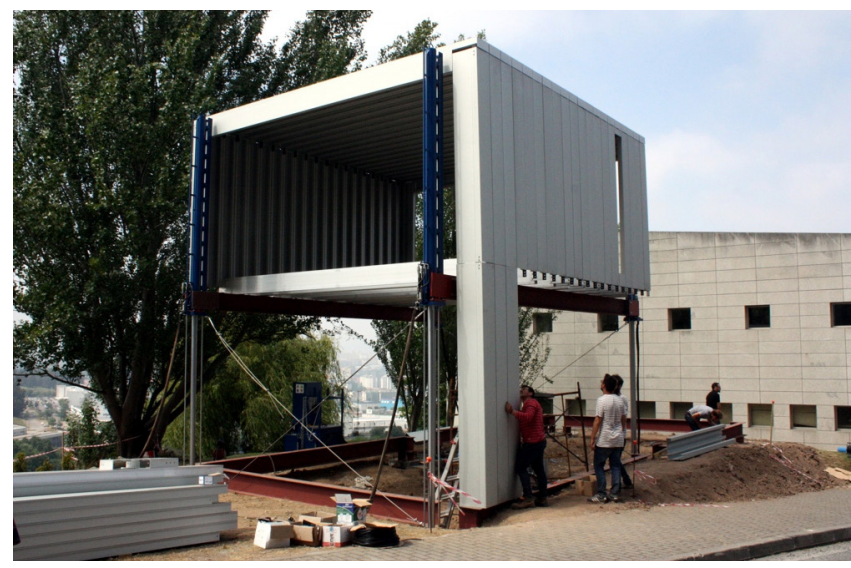

Figura 17. Construcción de la planta inferior.

sólo se pretendía construir dos niveles, con esta fase se dio el proceso como concluido. No obstante se aprovechó para izar el conjunto completo un nivel más sin que se apreciara problema alguno. A continuación se trasladaron los gatos al módulo de acero y se repitió el proceso completo para este segundo módulo.

Se continuó con el proceso montando un nuevo forjado apoyado en la cimentación perimetral y los paneles portantes de fachada. Este proceso se puede repetir tantas veces como plantas tenga el edificio. En este caso se construyeron sólo dos plantas, pero también se han elevado una vez construidas para comprobar la validez del sistema simplificado para edificaciones de hasta tres plantas (figura 18). Los cálculos teóricos indican que el número de plantas que pueden ser izadas con este sistema sería mayor, pero se deseaba hacer una comprobación experimental, al menos en los límites existentes.

\section{CONCLUSIONES}

De los estudios teóricos realizados y de la comprobación experimental, se deduce que el sistema Revestop es viable técnicamente. El proyecto REVERSTOP fue un proyecto interdisciplinar en el que participaron un equipo de construcción y otro de estructuras. Este artículo se refiere específicamente a los condicionantes estructurales, pero se diseñaron diversos tipos de paneles con un estudio minucioso de las condiciones térmicas y otros condicionantes funcionales, cumpliendo las condiciones exigidas por el Código Técnico en sus DB, que han sido tenidos en cuenta como parte fundamental del diseño del prototipo y se han contrastado con mediciones in situ.

Desde el punto de vista estructural se ha visto que se pueden resolver todos los elementos resistentes con un único perfil y que además sirve de piel exterior del edificio. Por otra parte consideramos que este sistema puede proporcionar un sistema constructivo más seguro que los habitualmente empleados y además permitir un diseño arquitectónico flexible, sin más condicionantes que la piel exterior resistente del edificio. Por otra parte al ser un sistema totalmen-

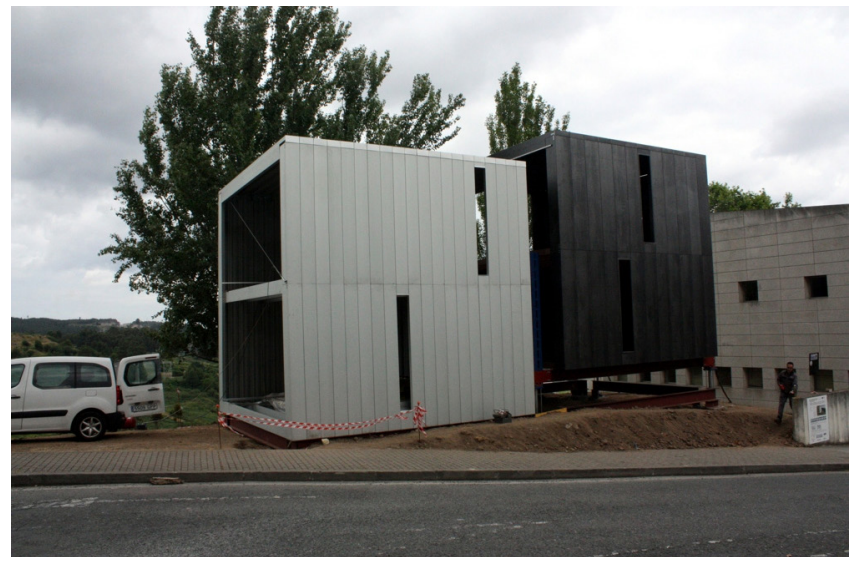

Figura 18. Izado final.

te desmontable, permite su reutilización las veces que sea necesario.

Los estudios realizados sobre los perfiles utilizados han probado su eficacia. En el caso de los perfiles de aluminio el límite a compresión es el que fija su capacidad resistente, pero es necesario un estudio de segundo orden. Con todo, el perfil diseñado sin refuerzos puede resolver adecuadamente edificaciones de hasta 6 plantas con luces de hasta 7,20 m. Entendemos que es un límite muy razonable. Edificaciones mayores precisarían gatos de más potencia. Es factible, pero se perdería la ligereza del sistema, que es una de sus características.

Se han analizado los elementos junta, de gran importancia en el diseño. Se han realizado ensayos de diferentes materiales, pero la conclusión alcanzada es que el más idóneo es el polietileno de alta densidad HDPE. Se han realizado ensayos a corto y largo plazo que avalan sus características resistentes.

Los resultados de esta investigación están siendo aplicados a un nuevo proyecto de investigación que está desarrollando nuestro equipo para el diseño de construcciones modulares y desplegables para situaciones de catástrofe humanitaria. Naturalmente el sistema citado supone la exigencia de un nivel tecnológico alto, pero las edificaciones se prevén para el alojamiento en países desarrollados de refugiados o naturales del país que hayan sufrido una catástrofe. Por ello es necesario disponer de sistemas de rápido montaje, aunque exijan medios tecnológicos más avanzados.

\section{AGRADECIMIENTOS}

Este documento ha sido posible gracias a una subvención de la XUNTA DE GALICIA a través de un proyecto de investigación (20). Los autores también desean agradecer la colaboración de Instalacións Parcero S.L.U., ALUTEC y Talleres Formoso en la realización del proyecto REVERSTOP. Igualmente desean agradecer a Guillermo Alonso y Silvia Miramontes del Laboratorio de Construcción de A Coruña EUAT su ayuda y colaboración en la realización de los ensayos de las juntas. 


\section{REFERENCIAS}

(1) Martínez Calzón, J.; Asensio Marchante, J. (1988). Elevación de la cubierta del Palau Sant Jordi. Revista de Obras Públicas. 135, (3275): 1099-1109.

(2) Vanderklaauw, P. M. (1974). Method of erecting a multi-story building and aparatus therefor. US Patent 3831902.

(3) Feld, Jacob; Carper, Kenneth L. (1997). Construction Failure (second ed.). New York: John Wiley \& Sons. pp. 311-315. ISBN 0-471-57477-5.

(4) Zallen, R. M.; Peraza, D. B. (2003). Engineering considerations for lift - slab construction. ASCE (American Society of Civil Engineers). https://doi.org/10.1061/9780784407059

(5) Masih, Rusk (1994). Stability of Lift Slab Structure During Construction Stage. Structures Congress XII 271-77. ASCE, 1994. http://cedb.asce.org/CEDBsearch/record.jsp?dockey=0086682.

(6) Briancon, Colbert. 2001. Procedimiento y dispositivo de realización semiautomática in situ, de depósitos metálicos. Spanish Patent. 2228855.

(7) UNE-EN 1993-1-3:2012. Eurocódigo 3: Proyecto de estructuras de acero. Parte 1-3: Reglas generales. Reglas adicionales para perfiles y chapas de paredes delgadas conformadas en frío. Recuperado de https://www.aenor.com/normas-ylibros/buscador-de-normas/une/?c=No049559

(8) Davies, J. M. (2000). Recent research advances in cold - formed steel structures. Journal of Construction Steel Research, 55(1-3): 267-288. https://doi.org/10.1016/So143-974X(99)ooo89-9

(9) Davies, J. M. (2006). Light gauge steel cassette wall construction - theory and practice. Journal of Constructional Steel Research, 62(11): 1077-1086. https://doi.org/10.1016/j.jcsr.2006.06.028

(10) Davies, J. M. (2007). Developments in stressed skin design. Thin-Walled Structures, 44(12): 1250-1260. https://doi. org/10.1016/j.tws.2007.01.002

(11) Davies, J. M.; Fragos, A. S. (2004). The local shear buckling of thin-walled cassettes infilled by rigid insulation. Journal of Constructional Steel Research, 60(3-5): 581-599. https://doi.org/10.1016/So143-974X(03)00131-7

(12) York, C. B.; Pedreschi, R. (2000). The influence of geometry on the strength of shear wall panels on light steel construction. Construction and Building Materials, 14(5): 277-285. https://doi.org/10.1016/So950-0618(00)00027-1

(13) Veljkovic, M.; Johansson, B. (2007). Light steel framing for residential buildings. Thin-Walled Structures, 44(12): 12721279. https://doi.org/10.1016/j.tws.2007.01.006

(14) Craveiro H.D.; Rodrigues J.P.C.; Laím L. (2016). Buckling resistance of axially loaded cold-formed steel columns. ThinWalled Structures, 106: 358-375. https://doi.org/10.1016/j.tws.2016.05.010

(15) Hermo Sánchez, Víctor Manuel. (2011). Sistema Constructivo Industrializado in Situ COTaCERO, (Tesis doctoral): University of A Coruña.

(16) Rodríguez Cheda, J.B.; Pérez- Valcárcel, J. ; Hermo, V. (2011). Método para construir edificaciones de varias plantas mediante paneles portantes ligeros desde el nivel del terreno. Spanish Patent. 2370438.

(17) Pérez-Valcárcel, J.; Hermo, V.; Rodríguez Cheda, J.B. (2013). A New Building System: Structural Aspects of CotaCero System. En Second International Conference On Structures \& Architecture. Guimarães, Portugal. pp 321-322. Publicación electrónica Structures and Architecture: Concepts, Applications and Challenges - Cruz (Ed.) (C) 2013 Taylor \& Francis Group, London, ISBN 978-0-203-79856-o pp 1056-1063.

(18) Pérez-Valcárcel, J.; Hermo, V.; Rodríguez Cheda, J.B. (2013). Structural Behaviour of CotaCero System during the Lifting Process. En International Conference Transformables 2013. Sevilla, España. 2013. pp 169-174. Escrig, F., y Sánchez J. (Ed.) Sevilla: Starbooks. ISBN 978-84-939565-3-0.

(19) Estévez Cimadevila, J.; Otero Chans, D.; Martín Gutierrez, E.; Vázquez Rodríguez, J. (2013) . Análisis experimental de las barras de madera laminada con sección tubular utilizadas en la construcción de una malla espacial. Maderas. Ciencia y Tecnología, 15(2): 153-170. https://doi.org/10.4067/So718-221X2013005000013

(20) Programa Conecta-Peme de la Xunta de Galicia (27-6-2014). Referencia IN852A 2014/12. 